\title{
La población y tasas vitales de las otras misiones jesuíticas de la Provincia de Paraguay y Moxos
}

\author{
The population and vital rates of the other Jesuit missions \\ of the Province of Paraguay and Moxos
}

Robert H. Jackson *

\section{Resumen}

Esta es la tercera y última de una serie de artículos que presentan información sobre la población y las tasas vitales de las misiones jesuíticas en la provincia de Paraguay reconstruidas a partir de censos. Este artículo discute las misiones de los Moxos que formaban parte de la Provincia de Perú, pero eran similares a los establecimientos Chiquitos. También se discuten las misiones de Tarima y Chaco, y la misión Jesús María de los Guenoas.

\section{Palabras claves}

Moxos, Tarima, Chaco, Guenoas, tasas vitales, población

\begin{abstract}
This is the third and last of a series of articles that present information on the population and vital rates of the Jesuit missions in the Paraguay province reconstructed from censuses. This article discusses the Moxos missions that were a part of the Peru Province but were similar to the Chiquitos establishments. It also discusses the Tarima and Chaco missions, and the short-lived mission Jesus Maria de los Guenoas.
\end{abstract}

\footnotetext{
*Investigador independiente, Ciudad de México. E-mail: robertvianey@gmail.com
} 


\section{Key Words}

Moxos, Tarima, Chaco, Guenoas, vital rates, population

Recibido: 15 de julio de 2017.

Evaluado: 4 de setiembre de 2017 . 
Este es el tercer artículo que analiza la población y las tasas vitales de las misiones jesuíticas de la Provincia de Paraguay ubicadas en lo que hoy son Brasil, Argentina, Paraguay y Bolivia Oriental. Los artículos anteriores discutieron las misiones de Chiquitos y las misiones establecidas entre los guaraníes, y este artículo trata de las otras misiones. El primer grupo son las misiones Moxos localizadas en Beni en el noreste de Bolivia. Aunque los jesuitas del Perú fundaron su grupo de misiones, se ubicaron cerca y eran similares a las misiones Chiquitos. Las misiones tenían poblaciones abiertas, es decir que los jesuitas reasentaron a un gran número de no cristianos en las misiones (Block, 1984: 80). También eran de alta fertilidad y alta mortalidad, pero experimentaron un crecimiento en los años de no crisis de mortalidad (Livi Bacci, 2016, p. 436).

El siguiente grupo son las dos misiones de Tarima: San Joaquín (establecida en 1746) y San Estanislao (establecida en 1749), situada al norte de las misiones guaraníes en lo que hoy es el Paraguay oriental. Los jesuitas registraron la población y el número de bautismos, matrimonios y entierros de las dos misiones en sus censos generales de las misiones guaraníes, y esta información permite reconstruir el perfil demográfico de las dos misiones. Eran poblaciones abiertas, lo que significaba que los jesuitas reasentaban a los no cristianos en las misiones. Los jesuitas no distinguían entre los bautismos de los no cristianos y los recién nacidos, por lo que las tasas de natalidad calculadas utilizando los censos están demasiadas altas. Además, la población de San Estanislao casi se duplicó en siete años, pasando de 1.090 en 1759 a 1.930 en 1766 . Este rápido crecimiento sólo fue posible con el reasentamiento de no cristianos. Asimismo, la población de San Joaquín aumentó de 1.046 en 1753 a 1.755 en 1765. En la mayoría de los años el número de bautismos fue mayor que el número de entierros resultando en el rápido aumento de la población. Sin embargo, hubo un exceso de enterramientos registrados en San Joaquín en 1765 causados muy probablemente por la viruela.

Jesús María de los Guenoas existió por treinta años (1690-1720) antes de que los jesuitas lo fusionaran con la misión de San Francisco de Borja tras una severa epidemia de viruela en 1718-1719. Los Guenoas eran un grupo Charrúa que vivía al este del río Uruguay. Después de la expansión jesuita en lo que hoy es Río Grande do Sul después de 1680 algunos Charrúa incluyendo Guenoas entraron en las misiones, mientras que otros continuaron atacando los colonos españoles. La población de Jesús María era una población abierta. Los jesuitas reasentaron a los no cristianos, adultos y niños, en la misión, lo que explica las fluctuaciones en el número de personas que viven en la misión y el gran número de bautismos en relación con la población. Los jesuitas registraron bautismos de niños recién nacidos y de no cristianos, tanto adultos como niños. Como tal, no es posible calcular las tasas exactas de natalidad.

El último grupo discutido aquí es las misiones del Chaco establecidas entre grupos como Abipones, Isistines, Mocovies y Tovas entre otros. Estos eran cazadores y recolectores nómadas, y las poblaciones congregadas en las misiones resultaron ser inestables. El registro más completo existe para San Fernando de Abipones, que es un censo que registró diez años consecutivos de población, bautismos, matrimonios y entierros. Los jesuitas bautizaban en su mayoría niños y pocos adultos. Aquellos adultos que aceptaron el bautismo generalmente lo hicieron a punto de morir. El bautismo y la aceptación del plan jesuita de ingeniería social habrían significado un cambio radical en su modo de vida, y particularmente un cambio en los papeles de trabajo de género.

La evidencia sugiere que los abipones permitieron el bautismo de sus hijos, lo que puede haber sido una condición impuesta por los jesuitas para su admisión en la comunidad misionera. Los jesuitas enterraron pocos adultos, y muy probablemente mu- 
rieron lejos de la misión. Un análisis de la estructura de edad y género de San Fernando de Abipones muestra que las mujeres y los niños constituían la mayoría de la población, y muchos hombres de Abipone eligieron no residir en la misión. Esto sugiere que Abipone utilizó la misión como lugar de refugio para las mujeres y los niños cuando los hombres iban a cazar o hacer guerra contra grupos indígenas rivales de colonos españoles. Su estatus se derivaba de sus habilidades como cazadores y guerreros, y el establecimiento de la misión como los agricultores habrían disminuido su prestigio. Un puñado de censos para otras misiones del Chaco muestran patrones similares de poblaciones inestables.

El objetivo de la política real en las fronteras era crear pueblos de indios estables similares a las comunidades indígenas en el centro de México y en la región andina. Sin embargo, este objetivo fracasó en muchos casos debido a las realidades demográficas y sociales de las poblaciones asentadas en las misiones. La misión como institución colonial logró un mayor grado de éxito en la creación de comunidades estables entre las poblaciones indígenas sedentarias, como los guaraníes, los diferentes grupos traídos a vivir en las misiones de Chiquitos, en las misiones de Tarima y en otras fronteras como Nuevo México y Sinaloa-Sonora en el norte de México. Las comunidades con poblaciones indígenas continuaron existiendo en los sitios de la misión después del abandono de los programas de la misión. Las poblaciones de cazadores-recolectores nómadas, por otra parte, demostraron ser demográficamente inestables y frágiles, y en algunos casos las poblaciones indígenas se enfrentaron a la extinción virtual cultural y biológica dentro de varias generaciones de entrar en las misiones. Esto ocurrió, por ejemplo, en las misiones de Baja California y las establecidas en Coahuila-Texas, ambas ubicadas en la frontera norte de México. Las misiones de los jesuitas del Chaco tenían perfiles demográficos similares, y es concebible que las poblaciones indígenas del Chaco hubieran experimentado un destino similar si los jesuitas hubieran podido convencerlos de aceptar la vida en las misiones y los cambios sociales que eso implicaba. Es este paradigma de las diferencias entre los grupos indígenas sedentario y no sedentario lo que explica la dinámica de los patrones demográficos en la provincia jesuita del Paraguay.

\section{Misiones Jesuíticas de Moxos}

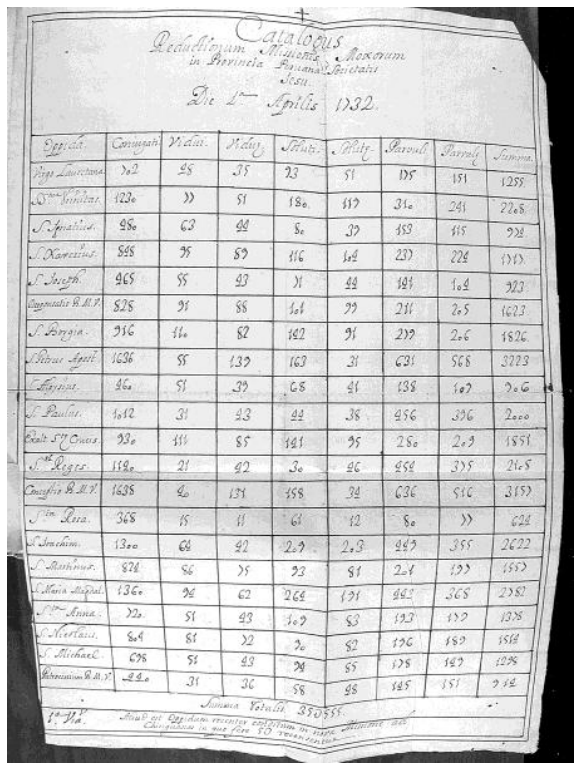

Censo de las misiones de Moxos en 1732. ARSI, Paraq. 13. 


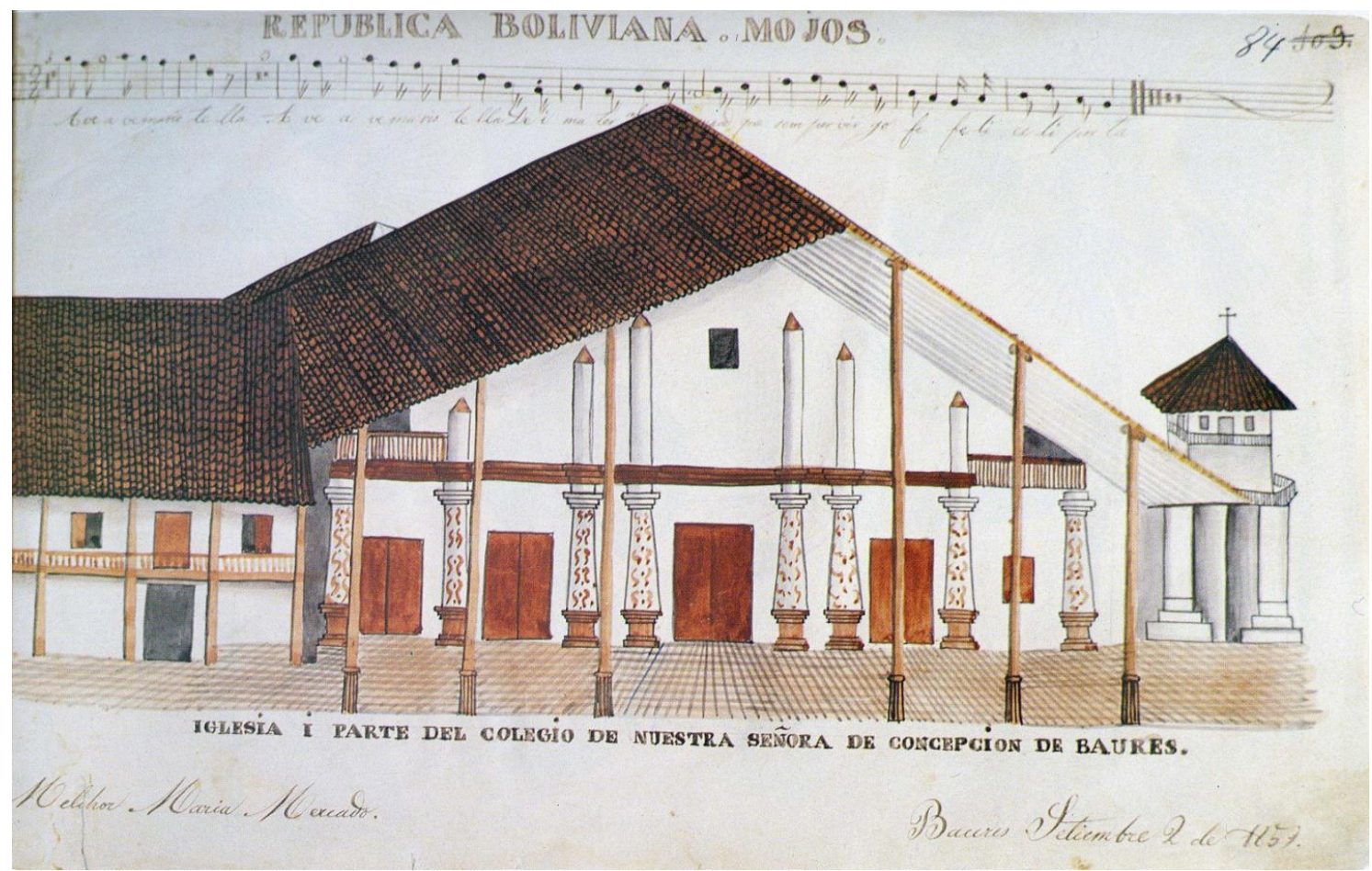

Concepción de Baures (Mercado, 1991) ${ }^{1}$.

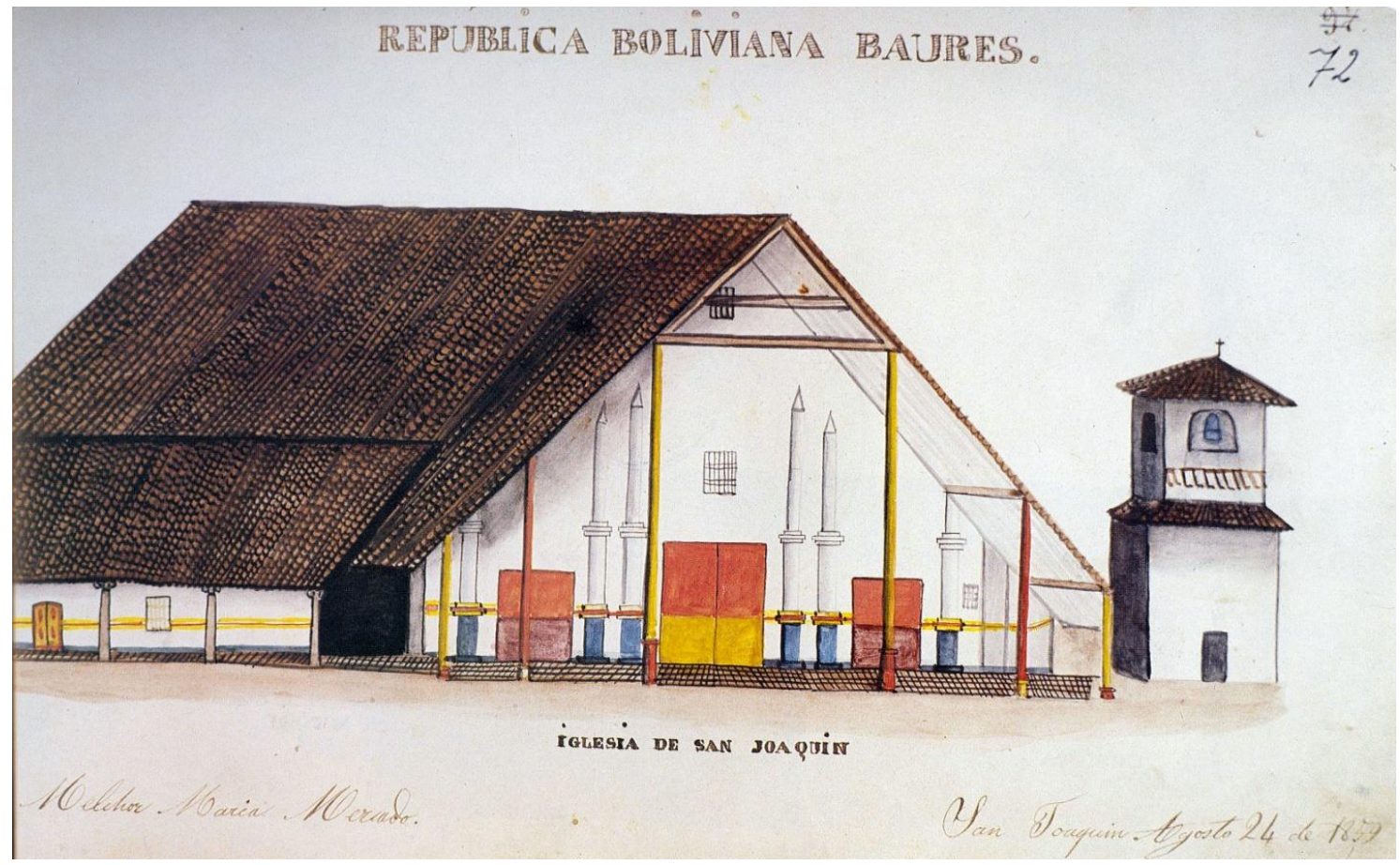

San Joaquín (Mercado, 1991).

\footnotetext{
${ }^{1}$ Melchor María Mercado (1816-1871) nació en Sucre, Alto Perú (Bolivia). Era abogado, funcionario público, explorador y pintor que documentó la sociedad de Bolivia entre 1840 y 1869 . El álbum original se encuentra en el Archivo Nacional de Bolivia en Sucre.
} 


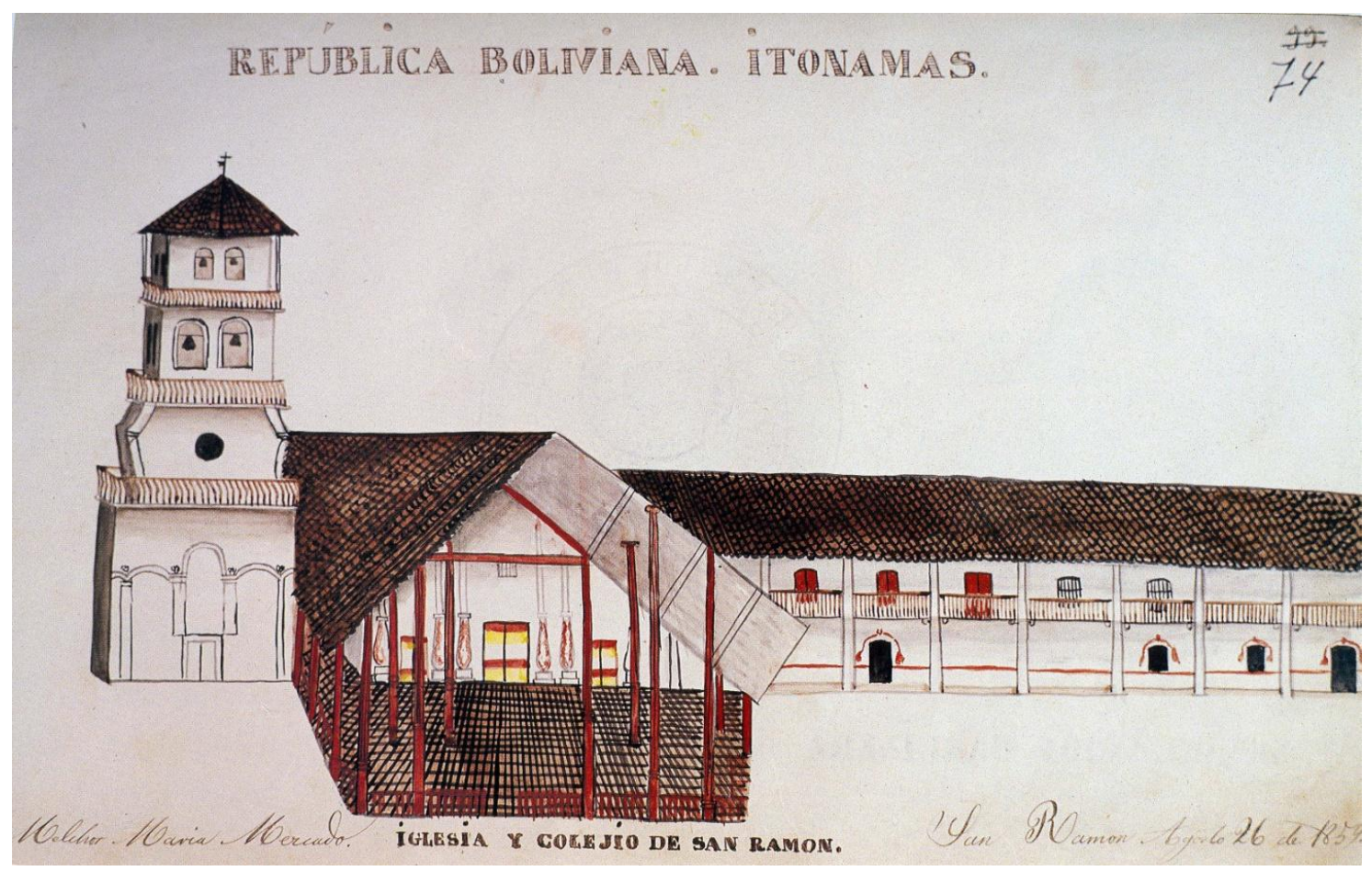

San Ramón (Mercado, 1991).

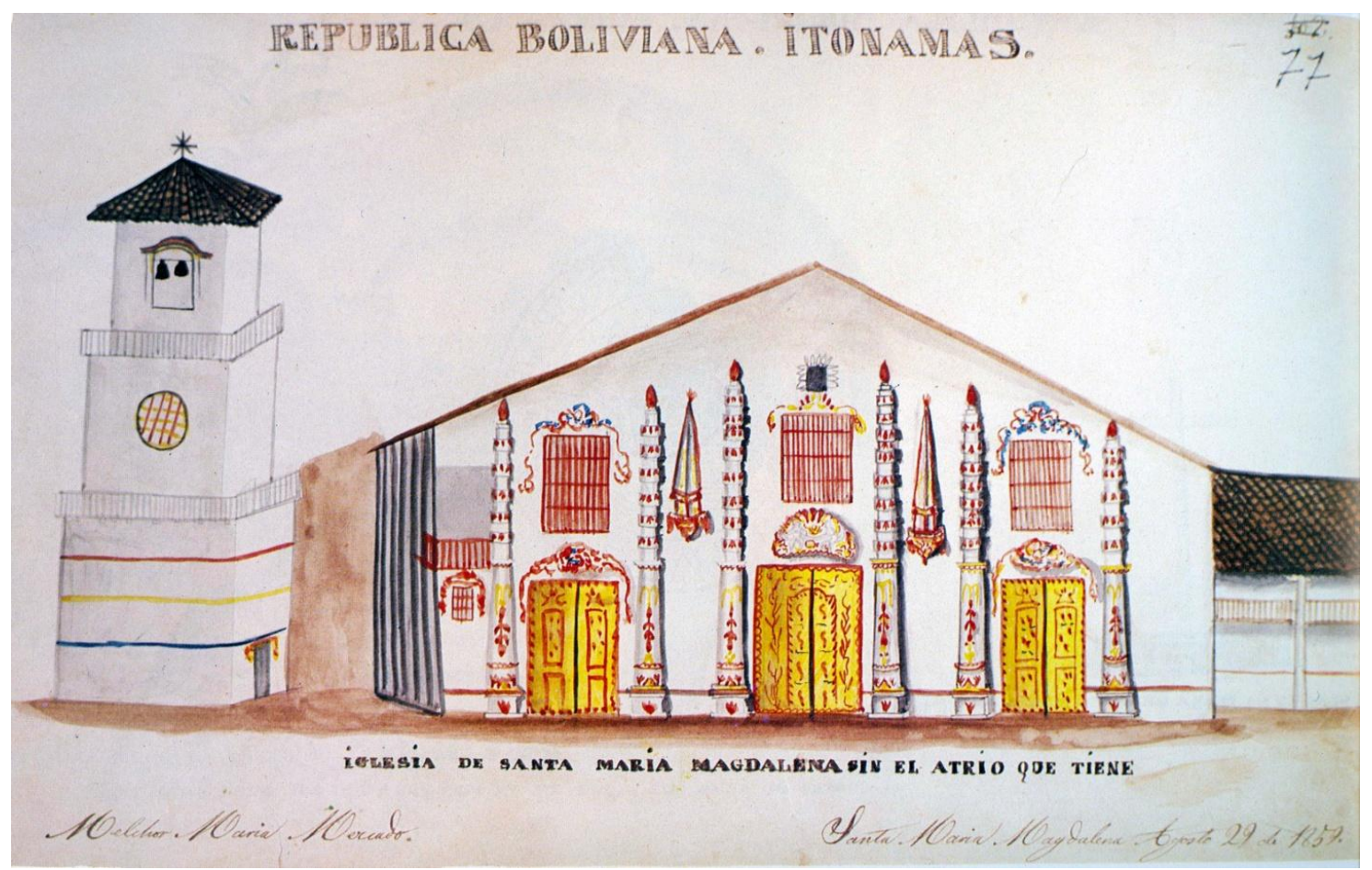

Santa María Magdalena (Mercado, 1991). 


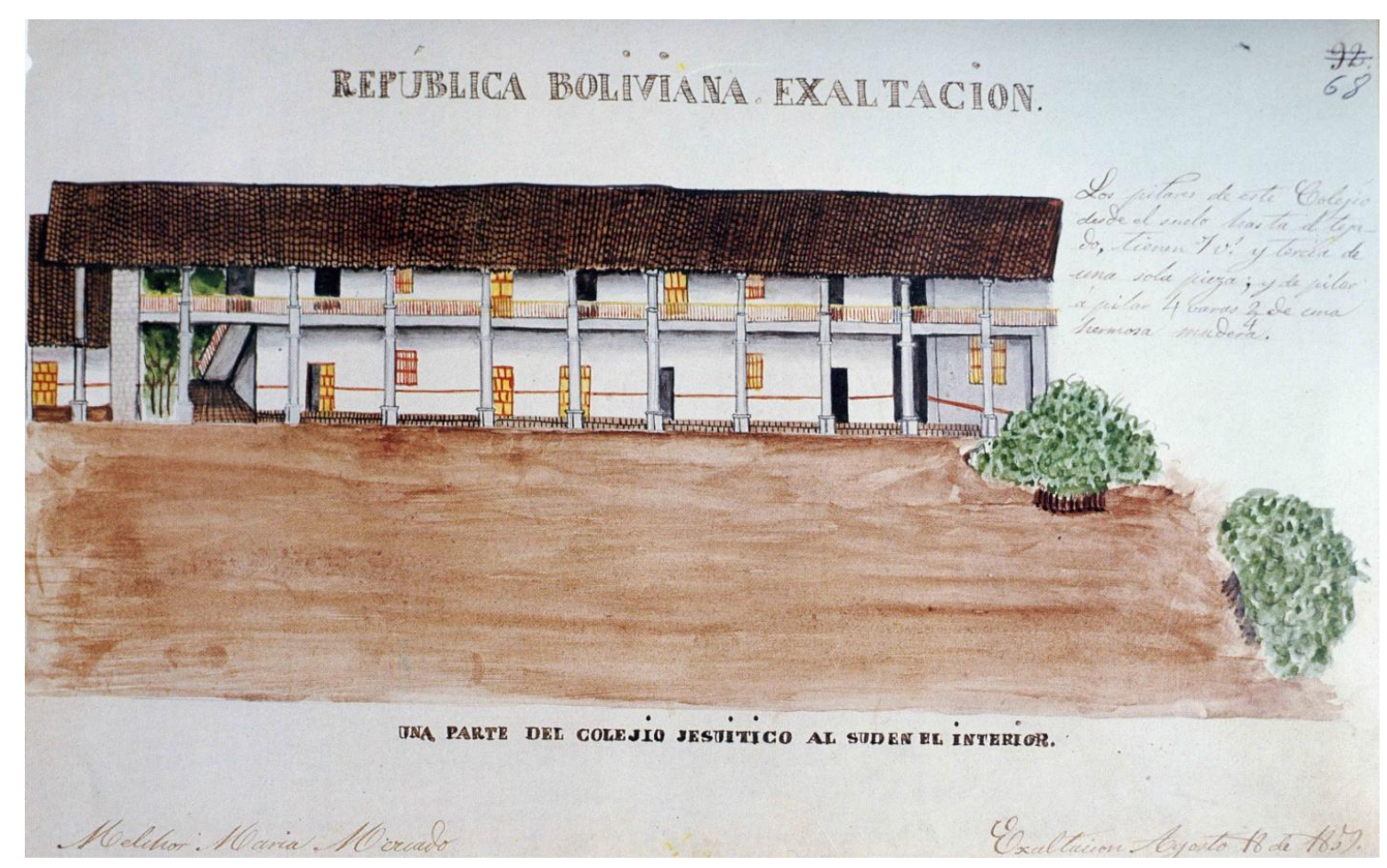

La Exaltación (Mercado, 1991).

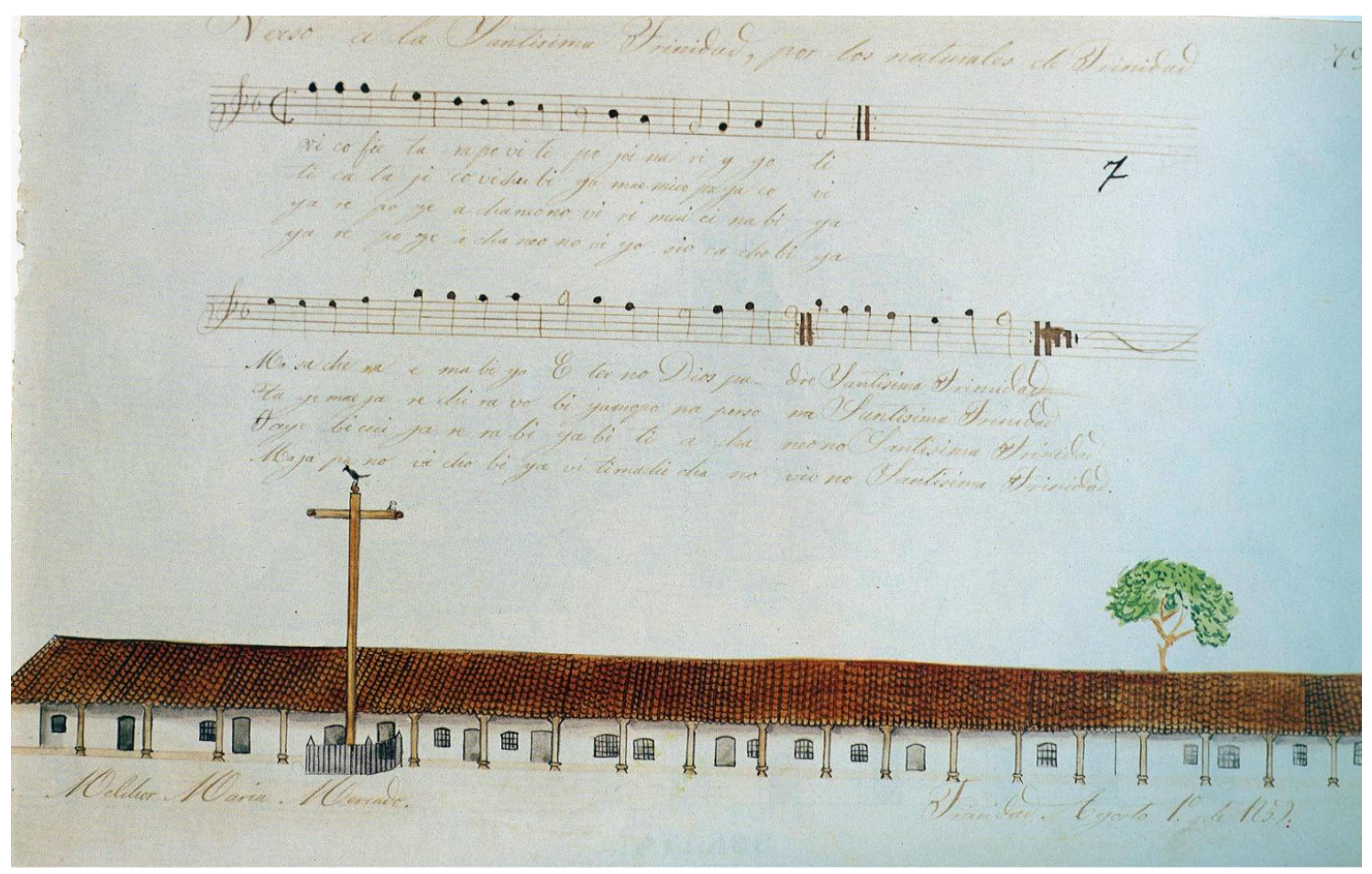

La Santísima Trinidad (Mercado, 1991). 
IHS. Antiguos Jesuitas en Iberoamérica ISSN: 2314-3908

\section{La Población de las Misiones de Moxos}

\begin{tabular}{|c|c|c|c|c|c|c|c|c|}
\hline Misión & Fundada & 1691 & 1698 & 1713 & 1717 & 1732 & 1748 & 1768 \\
\hline Trinidad & 1686 & 2254 & 2693 & 1700 & & 2208 & 1720 & 700 \\
\hline Esposorio & 1694 & & & & 1576 & 1623 & 1199 & \\
\hline Santa Rosa & 1705 & & & 1600 & 666 & 624 & 388 & \\
\hline Loreto & 1682 & 3822 & & 2000 & 1768 & 1255 & 1054 & 850 \\
\hline San Xavier & 1691 & 2361 & 1863 & 2000 & & 1717 & 1710 & 1200 \\
\hline San Pedro & 1697 & & & 1900 & 2864 & 3223 & 3296 & 2100 \\
\hline Exaltación & 1709 & & & 1400 & 1684 & 1851 & 1593 & 1900 \\
\hline San Ignacio & 1689 & 3014 & 3202 & 1505 & 1981 & 974 & 621 & 1160 \\
\hline San José & 1691 & 2036 & & 2105 & 1008 & 923 & 686 & \\
\hline San Miguel & 1696 & & & & & 1298 & 3444 & \\
\hline $\begin{array}{l}\text { S. L. Gon- } \\
\text { zaga }\end{array}$ & 1698 & & & 1630 & 1011 & 906 & 523 & \\
\hline S.F. Borja & 1693 & & & 1924 & 1256 & 1826 & 998 & \\
\hline San Pablo & 1703 & & & 1390 & 2048 & 2000 & 1324 & \\
\hline Los Reyes & 1710 & & & 1500 & 1641 & 2108 & 1782 & 1060 \\
\hline San Juan & 1710 & & & 1304 & 581 & & & \\
\hline Concepción & 1708 & & & & 2152 & 3157 & 2803 & 1295 \\
\hline San Joaquín & 1708 & & & 1206 & 2310 & 2622 & 2112 & 680 \\
\hline Santa Ana & 1709 & & & 200 & & 1378 & 1394 & 1000 \\
\hline Magdalena & 1720 & & & & & 2782 & 3112 & 4300 \\
\hline San Martin & 1717 & & & & & 1557 & 1222 & 600 \\
\hline San Nicolás & 1740 & & & & & 1514 & 1816 & 400 \\
\hline San Simón & 1744 & & & & & 914 & 493 & \\
\hline
\end{tabular}

Fuente: Catalogus Redoctionum Misionis Moxorum in Provincia Peruana Jesu Die 1 Aprilis 1732, Archivo Histórico de la Compañía de Jesús, Pastells, 1916, VI, pp. 157-158 y VII, pp. 746-748. Querejazu, 1995, p. 336.

\section{Misiones Jesuíticas de Tarima}

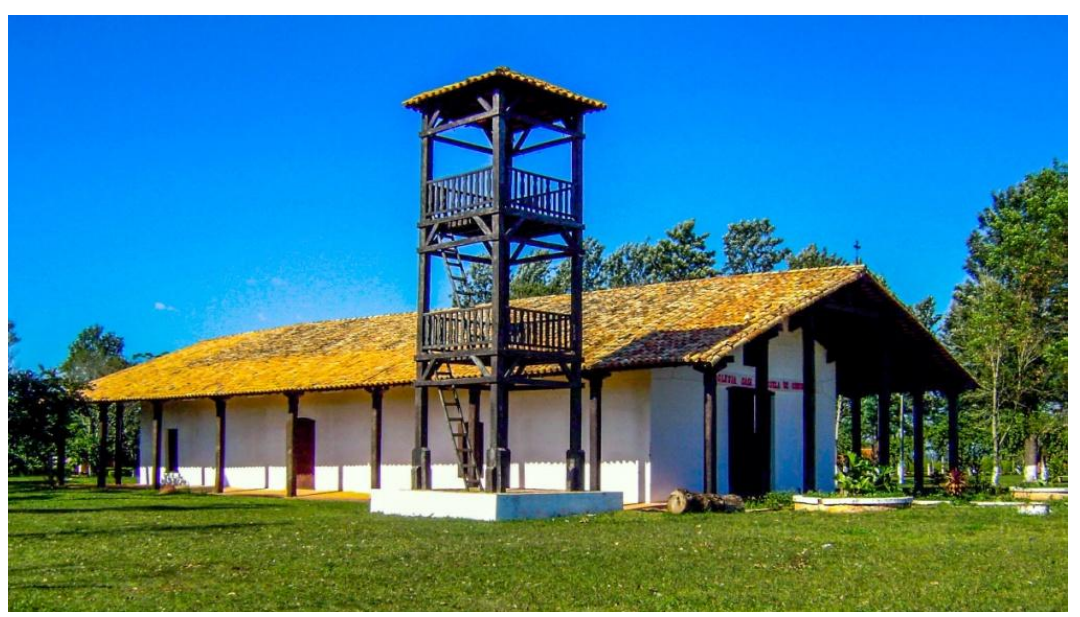

San Joaquín

111 Robert H. Jackson. La población y tasas vitals de otras misiones jesuíticas... 104-118. 


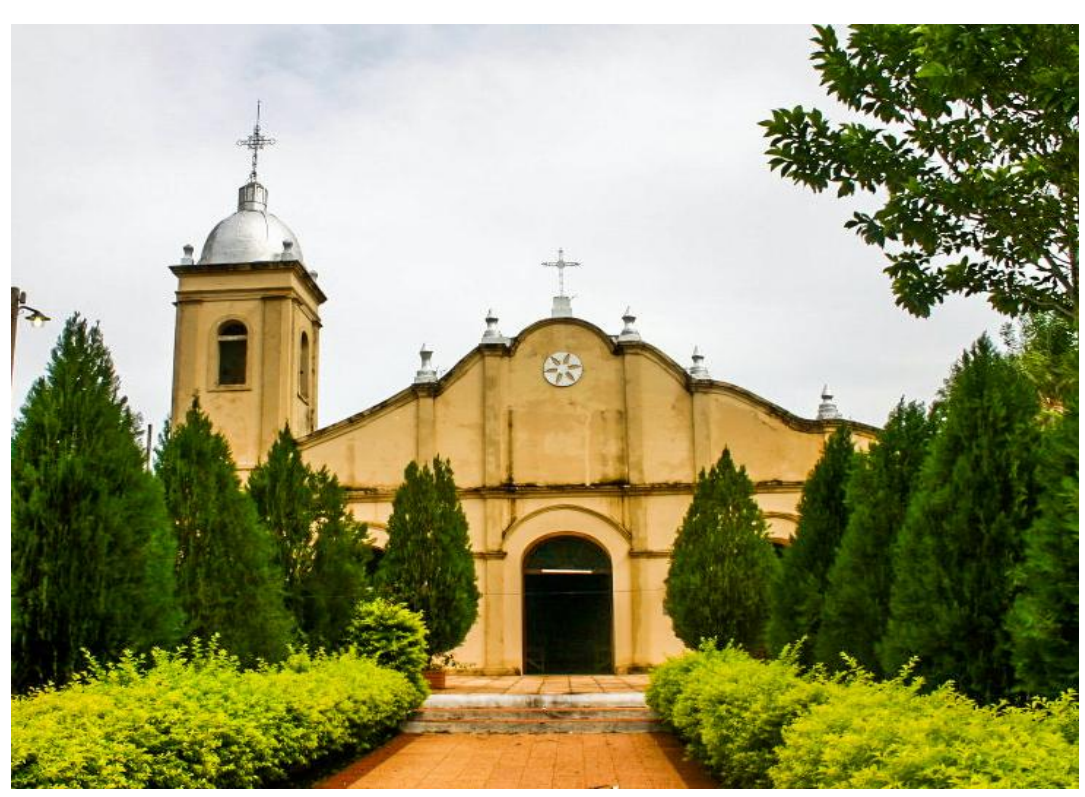

San Estanislao

La Población y Tasas Vitales de San Joaquín y San Estanislao

\begin{tabular}{|c|c|c|c|c|c|c|c|}
\hline Año & Población & Familias & Bautismos & Entierros & $\begin{array}{l}\text { Tasa } \\
\text { Bruta } \\
\text { Natalidad }\end{array}$ & $\begin{array}{l}\text { Tasa Bruta } \\
\text { Mortalidad }\end{array}$ & TPF $^{* *}$ \\
\hline \multicolumn{8}{|c|}{ San Joaquín } \\
\hline 1750 & 702 & & & & & & \\
\hline 1752 & 1052 & 208 & & & & & 5.1 \\
\hline 1753 & 1046 & 200 & 35 & 32 & 33.3 & 30.4 & 5.2 \\
\hline 1754 & 1088 & 200 & 73 & 16 & 69.8 & 15.3 & 5.4 \\
\hline 1755 & 1074 & 206 & & & & & 5.2 \\
\hline 1757 & 1112 & 222 & & & & & 5.0 \\
\hline 1759 & 1280 & 267 & 99 & 36 & $81.4^{*}$ & $28.1^{*}$ & 5.2 \\
\hline 1760 & 1287 & 270 & & & & & 4.8 \\
\hline 1762 & 1415 & 268 & 84 & 30 & $66.8^{*}$ & $23.9^{*}$ & 5.3 \\
\hline 1763 & 1707 & 355 & 94 & 85 & 66.4 & 60.1 & 4.8 \\
\hline 1764 & 1842 & 363 & & & & & 5.1 \\
\hline 1765 & 1755 & 372 & 119 & 152 & 64.6 & 82.5 & 4.7 \\
\hline 1766 & 1440 & 388 & 143 & 74 & 81.5 & 42.2 & 3.7 \\
\hline \multicolumn{8}{|c|}{ San Estanislao } \\
\hline 1750 & 735 & & & & & & \\
\hline 1753 & 808 & 137 & 62 & 18 & $76.3^{*}$ & $22.1^{*}$ & 5.9 \\
\hline 1754 & 600 & 180 & & & & & 3.3 \\
\hline 1755 & 835 & 150 & & & & & 5.6 \\
\hline 1756 & 503 & 110 & & & & & 4.6 \\
\hline 1757 & 941 & 223 & & & & & 4.2 \\
\hline 1759 & 1090 & 234 & 230 & 68 & $247.8^{*}$ & $73.3^{*}$ & 4.7 \\
\hline 1760 & 943 & 240 & & & & & 3.9 \\
\hline
\end{tabular}

112 Robert H. Jackson. La población y tasas vitals de otras misiones jesuíticas... 104-118. 
IHS. Antiguos Jesuitas en Iberoamérica ISSN: 2314-3908

Vol. 6 no 1

enero-junio 2018

\begin{tabular}{|l|l|l|l|l|l|l|l|}
\hline 1762 & 1182 & 279 & 210 & 42 & $217.2^{*}$ & $43.4^{*}$ & 4.3 \\
\hline 1763 & 1429 & 321 & 209 & 110 & 176.8 & 93.1 & 4.5 \\
\hline 1764 & 1589 & 360 & & & & & 4.4 \\
\hline 1765 & 1730 & 383 & 180 & 87 & 113.3 & 54.6 & 4.5 \\
\hline 1766 & 1930 & 415 & 149 & 37 & 86.1 & 21.4 & 4.7 \\
\hline
\end{tabular}

*Estimado **TPF -Tamaño Promedio de la Familia.

Fuente: (Jackson, 2015, p. 127).

La Población de San Joaquín, 1750-1799

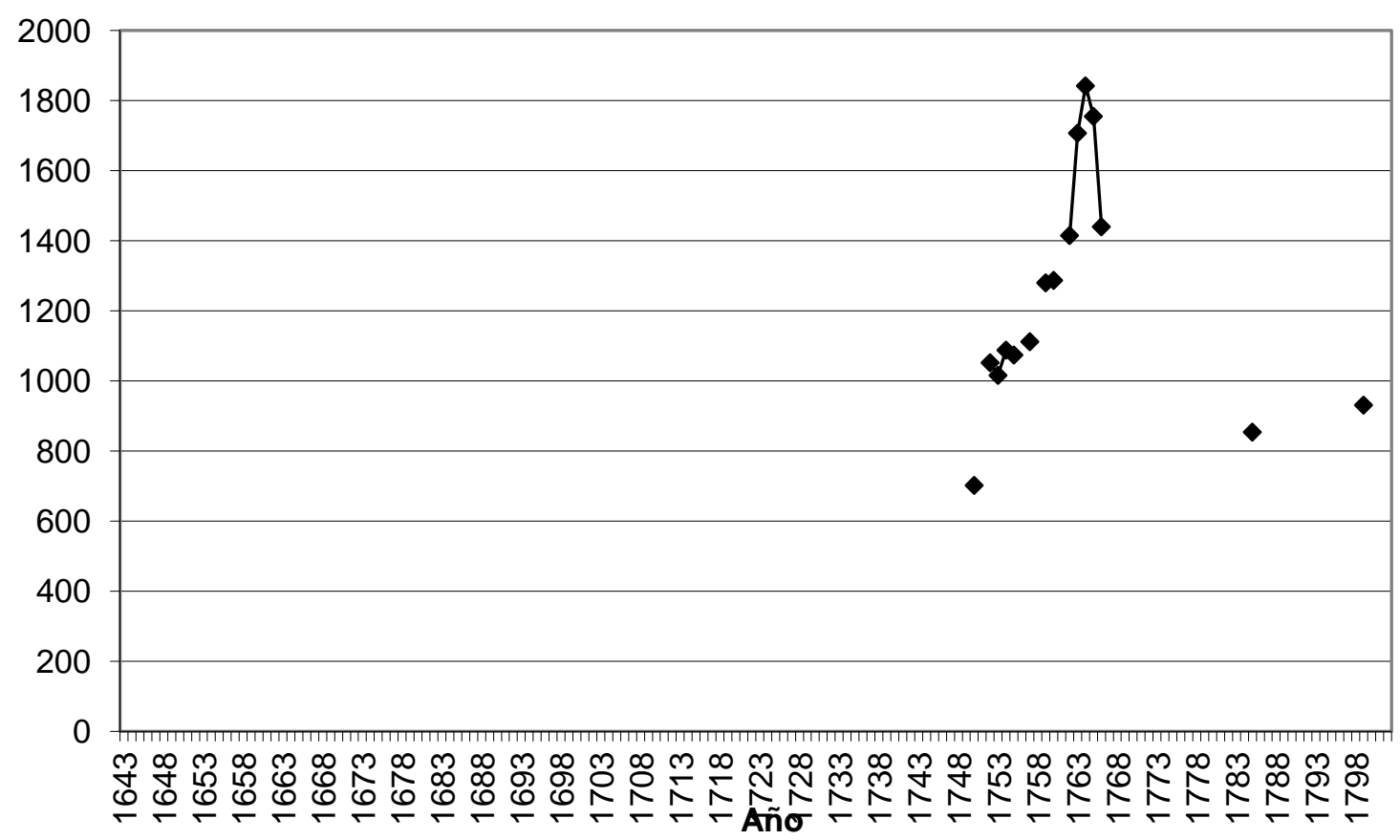

La Población de San Estanislao, 1750-1799

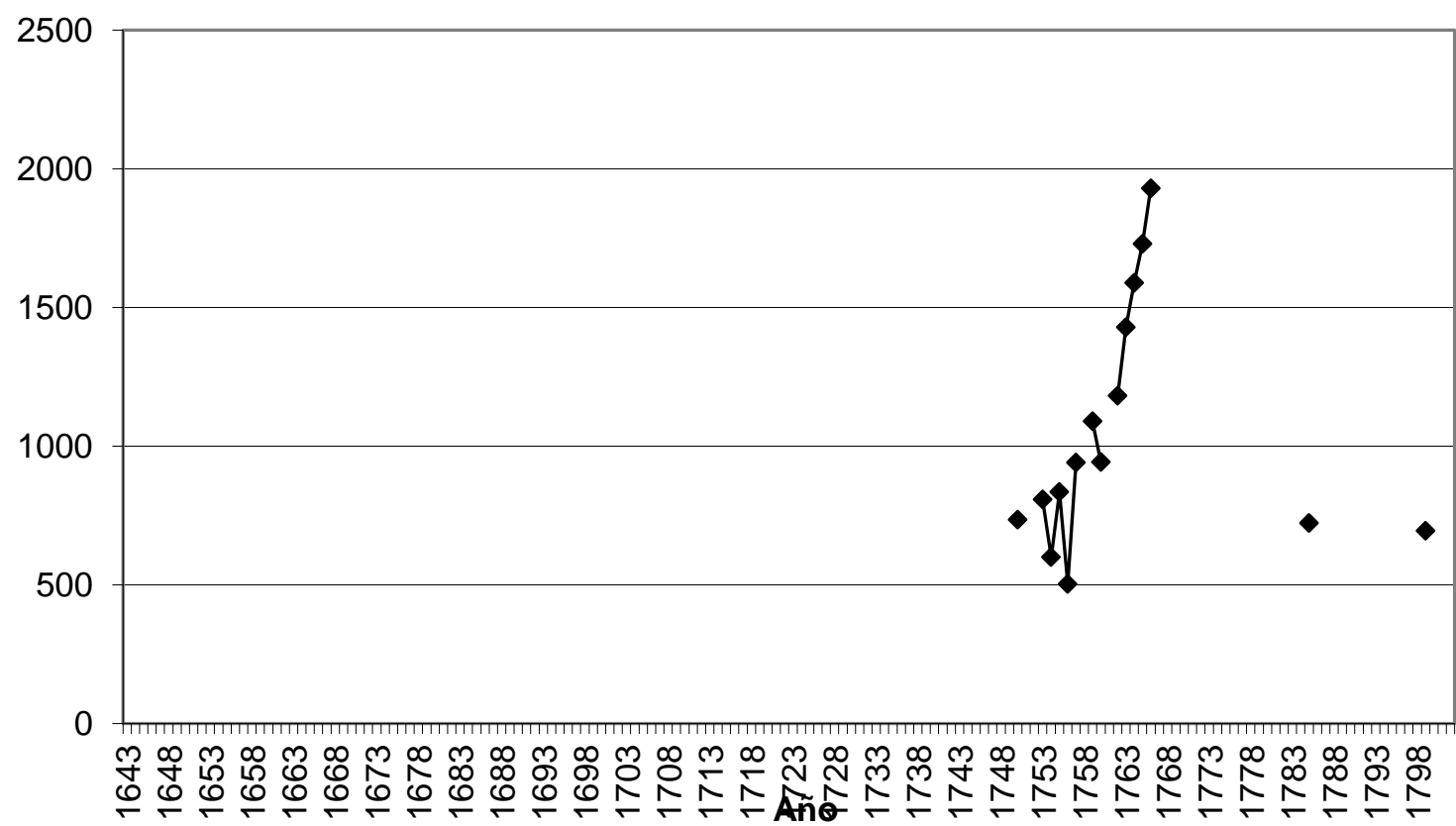

113 Robert H. Jackson. La población y tasas vitals de otras misiones jesuíticas... 104-118. 
IHS. Antiguos Jesuitas en Iberoamérica ISSN: 2314-3908

La Población y tasas vitales de Jesús María de los Guenoas

\begin{tabular}{|l|l|l|l|l|l|l|l|}
\hline Año & Población & Familias & Bautismos & Entierros & $\begin{array}{l}\text { Tasa } \\
\text { Bruta de } \\
\text { Natalidad }\end{array}$ & $\begin{array}{l}\text { Tasa Bruta } \\
\text { de } \\
\text { Mortalidad }\end{array}$ & TPF** \\
\hline 1690 & 334 & 74 & 51 & 38 & $158.7^{*}$ & $118.4^{*}$ & 4.5 \\
\hline 1691 & 300 & 74 & $30^{\wedge}$ & 26 & 87.8 & 77.8 & 4.1 \\
\hline 1694 & 298 & 89 & 44 & 41 & $149.2^{*}$ & $139^{*}$ & 3.4 \\
\hline 1698 & 200 & 80 & 30 & 24 & $154.6^{*}$ & $123.7^{*}$ & 2.5 \\
\hline 1702 & 200 & 79 & 32 & 29 & $162.4^{*}$ & $147.2^{*}$ & 2.5 \\
\hline 1705 & 288 & 97 & 41 & 17 & $155.3^{*}$ & $64.4^{*}$ & 3.0 \\
\hline 1708 & 303 & 98 & 34 & 36 & $111.5^{*}$ & $118.0^{*}$ & 3.1 \\
\hline 1714 & 357 & 140 & & & & & 2.6 \\
\hline 1715 & 281 & 67 & & & & & 4.2 \\
\hline 1716 & 307 & 71 & & & & & 4.3 \\
\hline 1717 & 283 & 85 & & & & & 3.3 \\
\hline 1719 & 238 & 51 & 25 & 16 & $109.2^{*}$ & $69.9^{*}$ & 4.7 \\
\hline
\end{tabular}

$\wedge^{\wedge}$ diez bautismos de adultos. *Estimado. **TPF -Tamaño Promedio de la Familia.

Fuente: Jackson, 2015, p. 247. “Anua numerazion de los Indios del Parana y Uruguay que estan a cargo de la Comp[añí]a de IESUS hecho a fin del año de 1691,” ARSI, Paraq. 12.

La población de Jesús María de los Guenoas, 1690-1719

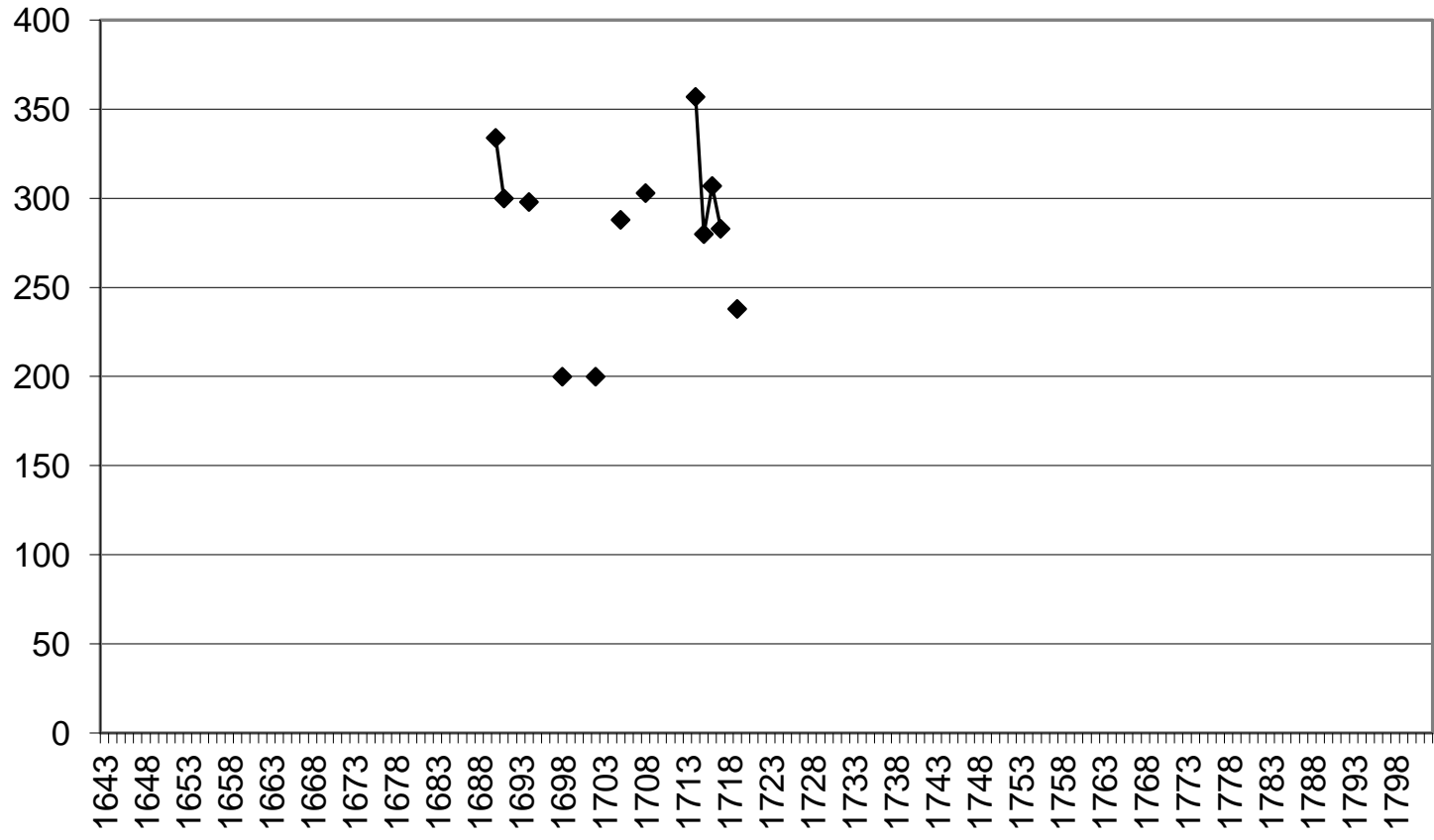

114 Robert H. Jackson. La población y tasas vitals de otras misiones jesuíticas... 104-118. 


\section{Misiones Jesuíticas del Chaco}

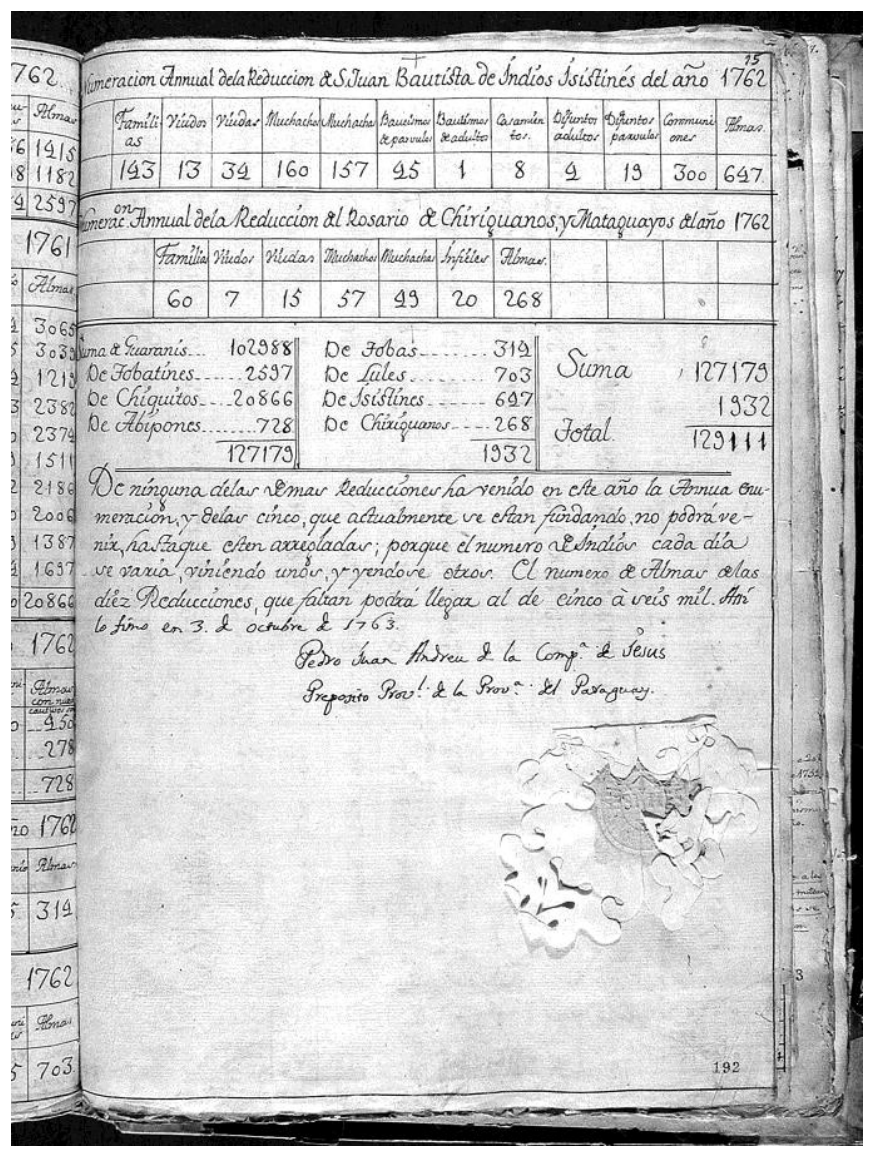

Censo de las misiones de Chaco en 1762. ARSI, Paraq. 13.

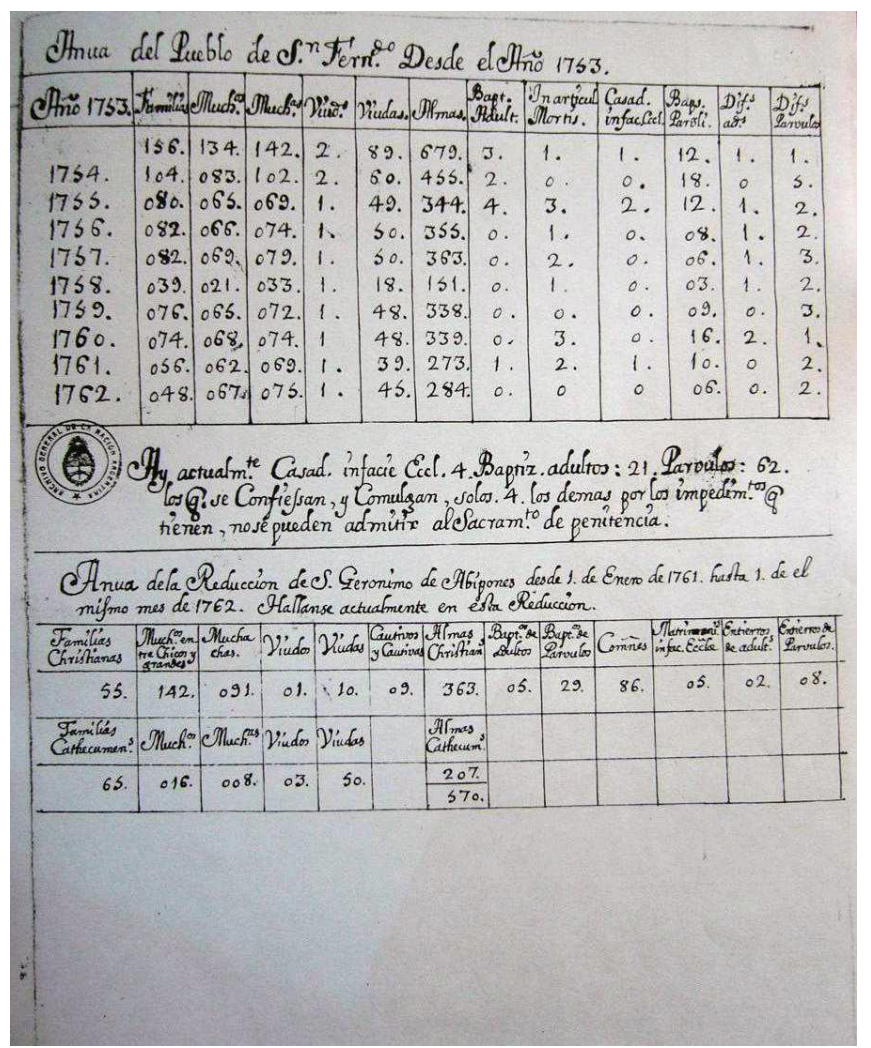

Censo de San Fernando de Abipones en 1762. AGN, Sala 9-10-6-10.

$115 \mid$ Robert H. Jackson. La población y tasas vitals de otras misiones jesuíticas... 104-118. 


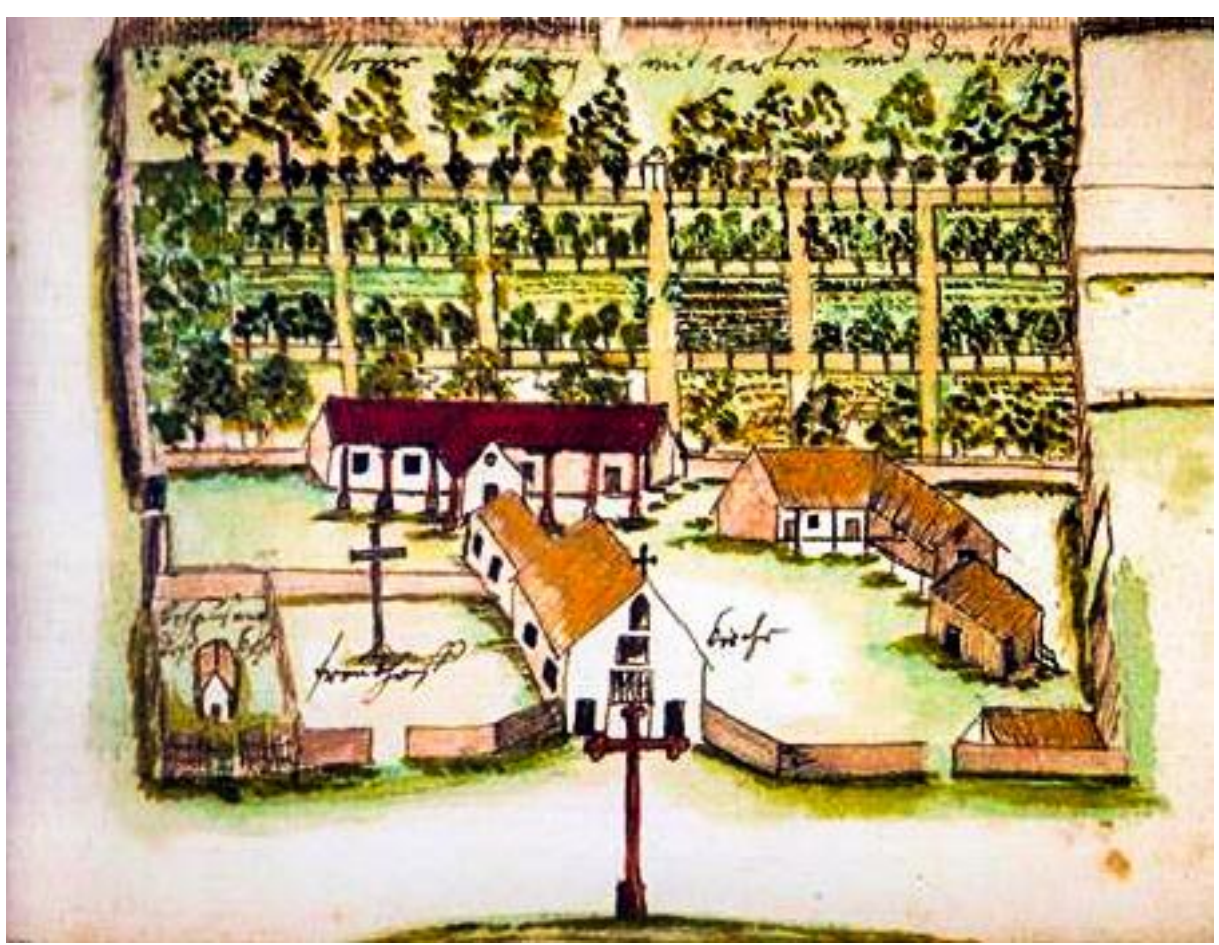

San Francisco Xavier de Mocovies. Por Florian Paucke, S.J.

\section{La población y tasas vitales de San Fernando de Abipones}

\begin{tabular}{|l|l|l|l|l|l|l|l|l|l|}
\hline & & & \multicolumn{2}{|l|}{ Bautismos } & & & & \\
\hline Año & $\begin{array}{l}\text { Pobla- } \\
\text { ción }\end{array}$ & $\begin{array}{l}\text { Fami- } \\
\text { lias }\end{array}$ & $\begin{array}{l}\text { Párvu- } \\
\text { los }\end{array}$ & $\begin{array}{l}\text { En } \\
\text { Articu- } \\
\text { Adul- } \\
\text { tos } \\
\text { Mortis }\end{array}$ & $\begin{array}{l}\text { Tasa } \\
\text { Bruta } \\
\text { Entie- } \\
\text { rros } \\
\text { dad }\end{array}$ & $\begin{array}{l}\text { Tasa } \\
\text { Bruta } \\
\text { Mortali- } \\
\text { dad }\end{array}$ & $\begin{array}{l}\text { TPF* } \\
*\end{array}$ \\
\hline 1753 & 679 & 156 & 12 & 3 & 1 & 2 & $17.9^{*}$ & $3.0 *$ & 4.4 \\
\hline 1754 & 455 & 104 & 18 & 2 & 0 & 5 & 26.5 & 7.4 & 4.4 \\
\hline 1755 & 344 & 80 & 12 & 4 & 3 & 3 & 26.4 & 6.6 & 4.3 \\
\hline 1756 & 355 & 82 & 8 & 0 & 1 & 3 & 23.3 & 8.7 & 4.3 \\
\hline 1757 & 363 & 82 & 6 & 0 & 2 & 4 & 8.5 & 11.3 & 4.4 \\
\hline 1758 & 151 & 39 & 3 & 0 & 1 & 3 & 8.3 & 8.3 & 3.9 \\
\hline 1759 & 338 & 76 & 9 & 0 & 0 & 3 & 59.6 & 19.9 & 4.5 \\
\hline 1760 & 339 & 74 & 16 & 0 & 3 & 3 & 26.6 & 8.9 & 4.6 \\
\hline 1761 & 273 & 56 & 10 & 1 & 2 & 2 & 29.5 & 5.0 & 4.9 \\
\hline 1762 & 284 & 48 & 6 & 0 & 0 & 2 & 22.0 & 7.3 & 5.9 \\
\hline
\end{tabular}

*Estimado. **TPF -Tamaño Promedio de la Familia.

Fuente: Jackson, 2015, p. 129. 


\section{La Población de las Misiones Jesuíticas del Chaco en 1762}

\begin{tabular}{|c|c|c|c|c|c|c|c|}
\hline \multirow[b]{3}{*}{ Misión } & \multicolumn{5}{|l|}{ Bautizados } & & \\
\hline & \multirow[b]{2}{*}{ Población } & \multirow[b]{2}{*}{ Familias } & \multicolumn{2}{|c|}{ Bautismos } & \multirow[b]{2}{*}{ Entierros } & \multicolumn{2}{|c|}{ No-Bautizados } \\
\hline & & & Adultos & Párvulos & & Población & Familias \\
\hline $\begin{array}{l}\text { San Jeró- } \\
\text { nimo } \\
\text { Abipones }\end{array}$ & 450 & 59 & 7 & 53 & 12 & 278 & 90 \\
\hline $\begin{array}{lr}\text { San } & \text { Este- } \\
\text { ban } & \text { de } \\
\text { Lules } \quad y \\
\text { Omoampa }\end{array}$ & 703 & 193 & 0 & 37 & 38 & 0 & 0 \\
\hline $\begin{array}{l}\text { San Igna- } \\
\text { cio Tobas }\end{array}$ & 314 & 67 & 6 & 31 & 22 & 0 & 0 \\
\hline $\begin{array}{l}\text { San Juan } \\
\text { Isistines }\end{array}$ & 647 & 143 & 1 & 45 & 23 & 0 & 0 \\
\hline Misión & $\begin{array}{l}\text { Tasa Bruta } \\
\text { Natalidad }\end{array}$ & $\begin{array}{l}\text { Tasa Bruta } \\
\text { Mortalidad }\end{array}$ & TPF** & & & & \\
\hline $\begin{array}{l}\text { San Jeró- } \\
\text { nimo } \\
\text { Abipones }\end{array}$ & $129.6^{*}$ & $29.3^{*}$ & 7.6 & & & & \\
\hline $\begin{array}{l}\text { San } \\
\text { ban } \\
\text { Lules } \\
\text { Omoampa } \\
\text { Omoam }\end{array}$ & $52.6^{*}$ & $54.0^{*}$ & 3.6 & & & & \\
\hline $\begin{array}{l}\text { San Igna- } \\
\text { cio Tobas }\end{array}$ & $101.6^{*}$ & $72.1^{*}$ & 4.7 & & & & \\
\hline $\begin{array}{l}\text { San Juan } \\
\text { Isistines }\end{array}$ & 72.0 & 36.8 & 4.5 & & & & \\
\hline
\end{tabular}

*Estimado **Tamaño Promedio de la Familia

Fuente: "Numeración Annual de la Reducción de S[an] Gerónimo de Abipones al año de 1762," ARSI, Paraq. 13; "Numeración Annual de la Reducción de S[an\} Estevan de la Nación Lule y Omoampa del año de 1762," ARSI, PARAQ. 13; "Numeración Annual de la Reducción de S[an] Ignacio de Indios Tobas del año de 1762," ARSI, PARAQ. 13; "Numeración Annual de la Reducción de S[an] Juan Bautista de Indios Isistines del año de 1762," ARSI, PARAQ. 13.

\section{La Población de las Misiones Jesuíticas del Chaco en Años seleccionados}

\begin{tabular}{|c|c|c|c|c|c|c|c|}
\hline \multirow[b]{3}{*}{ Misión } & \multicolumn{5}{|l|}{ Bautizados } & & \\
\hline & & & Bautism & & & \multicolumn{2}{|c|}{ No-Bautizados } \\
\hline & Población & Familias & Adultos & Párvulos & Entierros & Población & Familias \\
\hline $\begin{array}{l}\text { SFX Mo- } \\
\text { covies } \\
(1755)\end{array}$ & 826 & 151 & 27 & 77 & 3 & 143 & 28 \\
\hline $\begin{array}{l}\text { San Jeró- } \\
\text { nimo } \\
\text { Abipones } \\
(1761)\end{array}$ & 450 & 59 & 7 & 53 & 12 & 278 & 90 \\
\hline San Juan & 694 & 164 & 3 & 49 & 23 & 0 & 0 \\
\hline
\end{tabular}


IHS. Antiguos Jesuitas en Iberoamérica ISSN: 2314-3908

\begin{tabular}{|c|c|c|c|c|c|c|c|}
\hline $\begin{array}{l}\text { Isistines } \\
\text { (1762) }\end{array}$ & & & & & & & \\
\hline $\begin{array}{l}\text { San Igna- } \\
\text { cio Tobas } \\
(1764)\end{array}$ & 357 & 65 & 3 & 25 & 10 & 0 & 0 \\
\hline Misión & $\begin{array}{l}\text { Tasa Bruta } \\
\text { Natalidad }\end{array}$ & $\begin{array}{l}\text { Tasa Bruta } \\
\text { Mortalidad }\end{array}$ & TPF** & & & & \\
\hline $\begin{array}{l}\text { SFX Mo- } \\
\text { covies }\end{array}$ & $102.4^{*}$ & $4.0^{*}$ & 5.5 & & & & \\
\hline $\begin{array}{l}\text { San Jeró- } \\
\text { nimo } \\
\text { Abipones }\end{array}$ & $129.6^{*}$ & $29.3^{*}$ & 7.6 & & & & \\
\hline $\begin{array}{l}\text { San Juan } \\
\text { Isistines }\end{array}$ & 75.7 & 35.6 & 4.2 & & & & \\
\hline $\begin{array}{l}\text { San Igna- } \\
\text { cio Tobas }\end{array}$ & $73.1 *$ & $29.2^{*}$ & 5.5 & & & & \\
\hline
\end{tabular}

*Estimado **Tamaño Promedio de la Familia

Fuente: “Anua del Pueblo de S[an] Xavier de la Nación Mocobi del Año de 1755," AGN, Sala 9-6-10-4; Anua de la Reduccion de S[an] Geronimo de Abipones desde 1 de Enero de 1761 hasta 1 de el mismo mes de 1762," AGN, Sala 9-6-10-6; "Cathalogo Annua del Pueblo de S[an] Juan Baut[ist]a de Indios Isistines del Año 1762," AGN, Sala 9-6-10-6; Annua de la Reducc[io]n de S[a]n Ignacio de Tovas. Año 1764," AGN, Sala 9-6-10-6.

\section{Bibliografia}

Block, David (1984). Mission Culture on the Upper Amazon: Native Tradition, Jesuit Enterprise and Secular Policy in Moxos, 1680-1880 (Lincoln: University of Nebraska Press).

Jackson, Robert H. (2005). Missions and Frontiers of Spanish America: A Comparative Study of the Impact of Environmental, Economic, Political, and Socio-Cultural Variations on the Missions in the Rio de la Plata Region and on the Northern Frontier of New Spain. Scottsdale: Pentacle Press.

(2015). Demographic Change and Ethnic Survival Among The Sedentary Populations On The Jesuit Mission Frontiers of Spanish South America, 16091803: The Formation and Persistence of Mission Communities in a Comparative Context (Leiden: Brill Academic Publishers).

Livi Bacci, Massimo (2016). "The Depopulation of Upper Amazonia in Colonial Times," Revista de Indias, LXXVI (267), pp. 419-448.

Mercado, Melchor María (1991). Álbum de paisajes, tipos humanos y costumbres de Bolivia, 1841-1869. Sucre: Banco Central de Bolivia/Archivo Nacional de Bolivia/Biblioteca Nacional de Bolivia.

Pastells SJ, Pablo (1912). Historia de la Compañía de Jesús en la Provincia del Paraguay. 9 vols. Madrid.

Querejazu, Pedro (ed.) (1995). Las misiones jesuiíticas de Chiquitos. La Paz. 